\title{
Octocorales de las aguas profundas del oriente de Venezuela*
}

\author{
Luis Ruiz ${ }^{1}$ \& Martín Rada ${ }^{1}$ \\ ${ }^{1}$ Estación de Investigaciones Marinas de Margarita (EDIMAR) \\ Fundación La Salle de Ciencias Naturales (FLASA), calle Colón, Punta de Piedras \\ Apdo. 144, Porlamar 6301, Isla de Margarita, Venezuela
}

\begin{abstract}
RESUMEN. Se analiza la fauna de octocorales de aguas profundas, mayores de $40 \mathrm{~m}$, de la zona oriental de Venezuela. El material fue colectado mediante inmersiones con SCUBA o con una draga de arrastre tipo Beam Trawl. Se recolectaron 55 ejemplares que fueron identificados, fotografiados y preservados. Se identificaron 15 especies pertenecientes a 13 géneros de las familias: Ellisellidae, Plexauridae, Gorgoniidae, Clavularidae, Anthothelidae, Nephtheidae, Kophobelemnidae y Renillidae. De las especies identificadas tres son nuevos registros para Venezuela: Neospongodes portoricencis, Clavularia sp. y Thesea guadalupensis de las familias Nephtheidae, Clavularidae y Plexauridae respectivamente. T. guadalupensis había sido reportada en las islas de Guadalupe y Carriacau, mientras que N. portoricencis fue registrada en las islas Barbados, Puerto Rico y Cuba, siendo una especie cosmopolita en aguas profundas. Se discute la importancia del conocimiento de esta fauna para su debido manejo y conservación, así como su posible relación con lugares de pesca más profundos.
\end{abstract}

Palabras clave: octocorales, aguas profundas, Neospongodes, Clavularia, Thesea, Venezuela.

\section{Deep-water octocorals off Eastern Venezuela*}

\begin{abstract}
Deep-water octocoral fauna exceeding $40 \mathrm{~m}$ was analyzed off Eastern Venezuela. The material was collected by SCUBA diving or with a Beam Trawl-type drag. In total, 55 specimens were collected, identified, photographed, and preserved. These included 15 species belonging to 13 genera of the following families: Ellisellidae, Plexauridae, Gorgoniidae, Clavularidae, Anthothelidae, Nephtheidae, Kophobelemnidae, and Renillidae. Three of the identified species are registered for the first time in Venezuela: Neospongodes portoricencis, Clavularia sp. and Thesea guadalupensis of the families Nephtheidae, Clavularidae, and Plexauridae, respectively. T. guadalupensis was reported for the islands of Guadalupe and Carriacau, and N. portoricencis, a cosmopolitan species in deep waters, for Barbados, Puerto Rico, and Cuba. The importance of this information for the proper management and conservation of the fauna is discussed, as are its possible relationships with deeper fishing sites.
\end{abstract}

Key words: octocorals, deep waters, Neospongodes, Clavularia, Thesea, Venezuela.

Autor corresponsal: Martín Rada (mrada@edimar.org)

\section{INTRODUCCIÓN}

El estudio de los octocorales del Caribe no ha correspondido, en intensidad y número de publicaciones, a la riqueza y variedad que estos componentes tienen en las comunidades arrecifales. Aunque en 1860 se publica el primer estudio faunístico de los corales de las Indias Occidentales, donde se incluyen a los octocorales (Duchassaing \& Michelotti, 1860), no es sino en la década del 60 cuando se realiza la primera revisión taxonómica exhaustiva de este grupo (Bayer, 1961) y sólo en los años 80, se puede encontrar un avance considerable en el número y diversidad de trabajos que se publicaron sobre este taxón en esta región del Atlántico (Botero, 1987; Dahlgren, 1989; Lewis \& Von Wallis, 1991; Keith, 1992; ver Romero \& Rada, 1999, para una revisión más exhaustiva de este punto).

* Trabajo presentado en el XXV Congreso de Ciencias del Mar de Chile y XI Congreso Latinoamericano de Ciencias del Mar (COLACMAR), realizados en Viña del Mar, entre el 16 y 20 de mayo de 2005. 
Para Venezuela en particular, se han citado de 47 (Losada \& Pauls, 2000) a 53 (Ramírez, 2001) especies de octocorales. Según Losada \& Pauls (2000), de las 47 especies citadas, 43 son gorgonáceos (6 familias y 16 géneros), dos telestáceos (1 familia, 1 género) y dos pennatuláceos (1 familia, 1 género). La revisión realizada por Ramírez (2001), ubica a 51 de las 53 especies citadas, como alcionáceos (6 familias y 19 géneros), y dos como pennatuláceos (2 familias y 2 géneros). A estas habría que agregar dos nuevos registros de gorgonáceos, pertenecientes a dos géneros no citados previamente (Rada \& Ruiz, 2002).

Entre los estudios efectuados en el país sobre esta fauna, se puede señalar el realizado por Álvarez (1981) sobre factores de mortalidad de los octocorales del Parque Nacional (PN) Morrocoy, Estado de Falcón; el de Fuenmayor (1983) sobre morfología funcional, crecimiento y distribución de octocorales en el PN Morrocoy y el de La Schiazza (1985) en el PN Archipiélago de Los Roques. Álvarez (1981) y Díaz (1983) estudian diversos parámetros comunitarios de los gorgonáceos en un bajo cercano a Cayo Sombrero en el PN Morrocoy. Márquez et al. (1997) reportan la distribución batimétrica de 26 especies de gorgonáceos en isla Alcatraz, en el PN San Esteban, Estado de Carabobo. Rada (1997) reporta la distribución, talla y densidad de dos especies de octocorales en un bajo cercano a Cayo Sombrero, en el PN Morrocoy. Rada \& Losada (1998) estudiaron la relación simbiótica entre un alga filamentosa y dos especies de gorgonáceos en el PN Morrocoy. Romero \& Rada (1999) estudian los octocorales de las aguas costeras de la isla de Margarita y Rada \& Ruiz (2002) analizan los octocorales de la isla de La Blanquilla.

En relación a los trabajos realizados sobre octocorales en la zona oriental de Venezuela, Deichmann (1936) publicó uno de los primeros estudios sobre la identificación de octocorales donde se incluye la zona oriental del país, específicamente en Cumaná. Stiasny (1941) identifica siete especies alrededor de las islas La Blanquilla y Los Frailes. Rodríguez (1959) encuentra tres especies para la isla de Cubagua y una para la laguna de Las Marites, isla de Margarita. Bayer (1961) señala una para el puerto de Guanta y una para el puerto de Cumaná. González (1970, 1972) indica 12 especies para la isla de Margarita y 10 especies para el golfo de Cariaco. Ramírez \& González (1974) indican la presencia de 11 especies para la isla de Coche. Ramírez (1978) realiza una caracterización general de las comunidades corali- nas, incluyendo los octocorales, presentes en la isla de Cubagua. En un estudio realizado en los islotes Caribe y Los Lobos, adyacentes al Morro de Chacopata, Estado de Sucre, Ramírez (1997) encontró 18 especies de octocorales. Romero \& Rada (1999) reportan 15 especies para la isla de Margarita y Rada \& Ruiz (2002) citan 22 para la isla de La Blanquilla. A pesar de esta diversidad de estudios, es muy escasa la información disponible sobre octocorales en aguas más profundas de esta región.

El presente trabajo tiene como objetivo identificar los octocorales de aguas profundas $(>40 \mathrm{~m})$, recolectados en la zona oriental de Venezuela.

\section{MATERIALES Y MÉTODOS}

En nueve campañas oceanográficas y visitas realizadas por investigadores de la Estación de Investigaciones Marinas de Margarita (EDIMAR) a diversas regiones costeras e insulares del oriente de Venezuela entre 1983 y 2004 (Fig. 1), se recolectaron especímenes de octocorales en 12 localidades con profundidades mayores a $40 \mathrm{~m}$, mediante inmersiones con equipo SCUBA (40-50 $\mathrm{m}$ de profundidad) y con dragas tipo Van Been de $0,19 \mathrm{~m}^{2}$ o tipo Beam Trawl de 1,8 $\mathrm{m}$ de ancho por $0,9 \mathrm{~m}$ de alto, ambas utilizadas a profundidades mayores de $50 \mathrm{~m}$, con el B/O "Hno. Gines" de la Fundación La Salle de Ciencias Naturales (FLASA). Después de registrar diferentes observaciones sobre las colonias en fresco (tamaño, color, forma de los pólipos si estaban extendidos, entre otros aspectos), los ejemplares de cada morfoespecie se preservaron tanto en seco como en húmedo con alcohol al 70\%.

En las campañas oceanográficas realizadas, cinco en la isla de La Blanquilla (1983, 1985, 1987, 1993, 1994), una al norte de la isla La Tortuga (2001), una al norte de la península de Paria (2000) y dos en la plataforma Deltana $(2003,2004)$, se recolectó un total de 55 especímenes (Fig. 1). Los ejemplares se identificaron utilizando las claves taxonómicas de Bayer $(1961,1981)$ y el trabajo clásico de Deichmann (1936). Para la preservación y preparación de sus escleritos se siguió la técnica descrita por Bayer (1961). Los individuos mejor conservados de cada especie se depositaron en la colección del Museo Oceanológico "Hermano Benigno Román" en Punta de Piedras, de la EDIMAR, siendo catalogados como MOBR I-\#. Adicionalmente, se realizó un registro fotográfico de cada ejemplar y muestras de sus principales tipos de escleritos utilizando una cámara digital y 


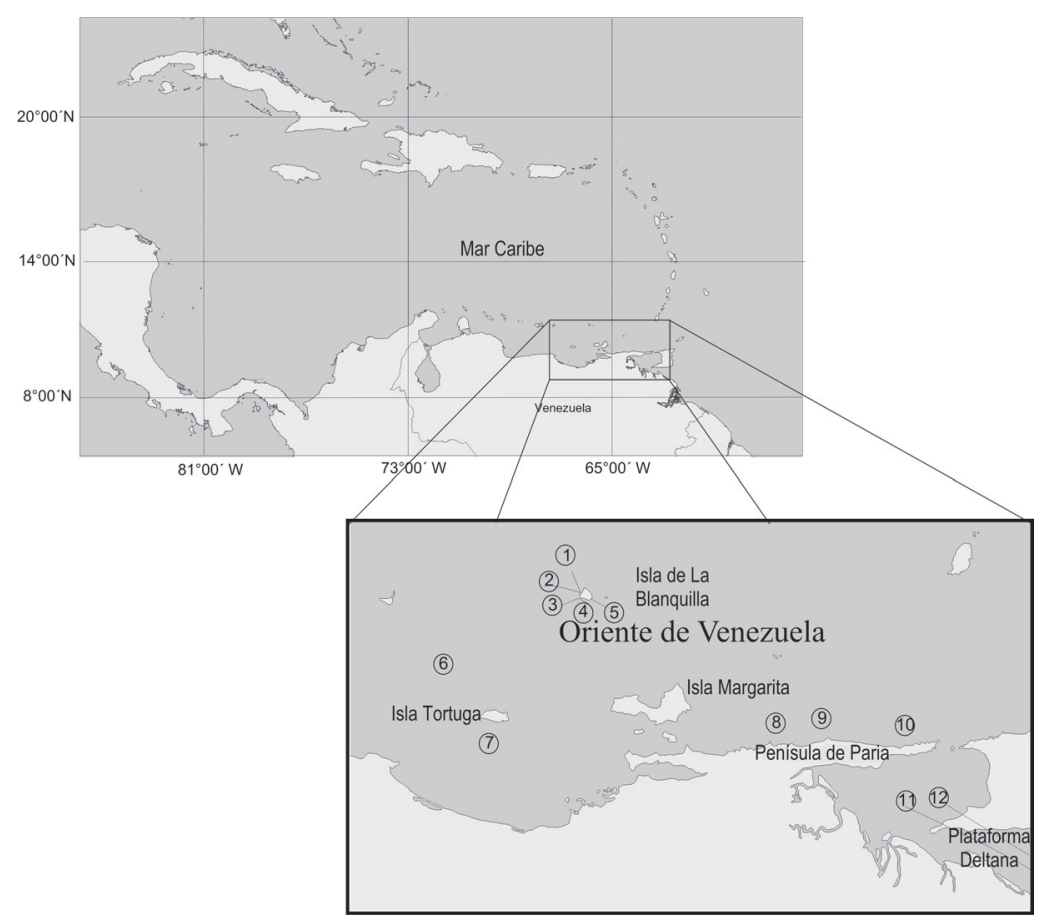

Figura 1. Área de estudio y localidades de muestreo.

Figure 1. Study area and sampling localities.

un microscopio óptico. Las imágenes se procesaron mediante el programa Adobe Photoshop 7,0 y Corel PhotoPaint 11,0.

\section{RESULTADOS}

Los 55 ejemplares de octocorales colectados fueron identificados como pertenecientes a 15 especies, ubicadas en 13 géneros de las familias: Ellisellidae, Plexauridae, Gorgoniidae, Clavularidae, Anthothelidae, Nephtheidae, Kophobelemnidae y Sessiliflorae.

A continuación, se presenta la ubicación taxonómica de dichas especies según Fabricius \& Alderslade (2001), una breve descripción de las mismas, así como el número de catálogo del MOBR asignado.

Subclase OCTOCORALLIA Haeckel, 1866

Orden ALCYONACEA Lamouroux, 1812 (Verrill, 1866)

\section{Grupo ALCYIONIINA}

Familia NEPHTHEIDAE Gray, 1862 (Utinomi, 1954)
Género Neospongodes Kükenthal, 1903 Neospongodes portoricencis Kükenthal, 1903 (Fig. 2).

Localidad: norte de la península de Paria (MOBR I-793).

Descripción: la colonia en general presenta las ramas cortas y robustas, que provienen de un mismo tallo. Los cálices son grandes y curvados hacia arriba, apoyados por un grupo de gruesas espinas convergentes. Opérculo que consiste en ocho pares de espinas curvas, generalmente desiguales y algunas barras. El color de las colonias en alcohol es crema claro y las espículas son translucidas.

Profundidad: $85 \mathrm{~m}$.

Sustrato: adherida a sustrato duro (restos de coral escleractinido).

\section{Grupo STOLONIFERA}

Familia CLAVULARIIDAE Hickson, 1894

Género Carijoa Müller, 1867

Carijoa riisei Duchassaing \& Michelotti, 1860.

Localidades: norte de la península de Paria (MOBR I-770, I-732) y sur de la isla La Tortuga (MOBR I-766). 
Descripción: colonia de $25 \mathrm{~cm}$ de longitud, las ramas parten de unos estolones y se desarrollan en forma aglutinada en forma arborescente. Las ramas tienen 5 a $10 \mathrm{~cm}$ de longitud, donde se notan ocho surcos extendidos a lo largo de ésta. Los cálices están proyectados y reticulados, su color es blanco al igual que toda la colonia.

Profundidad: 50 a $130 \mathrm{~m}$.

Sustrato: fondo de cascajos y restos de corales.

Género Clavularia Blainville, 1830

\section{Clavularia sp. (Fig. 3)}

Localidad: sur de la isla La Tortuga (MOBR I768).

Descripción: colonia con solo una rama de color blanco. Los cálices tienen más de $2 \mathrm{~cm}$ de color naranja oscuro en su borde y están adosados al eje principal. En un corte transversal se observan los canales que están paralelos a la colonia atravesándola en toda su extensión, los escleritos externos de la colonia son en su mayoría husos que van desde 0,08 a 0,55 $\mathrm{mm}$ de largo. Los escleritos que se encuentran en el borde del cáliz, son husos alargados de $0,1 \mathrm{~mm}$ de largo y anaranjados. El antocodio está fuertemente armado con husos de 0,5 a 0,6 $\mathrm{mm}$ de largo, presenta una corona con husos arqueados y los tentáculos los conforman ocho grandes formaciones de escleritos con forma de husos de $0,01 \mathrm{~mm}$ de longitud.

Profundidad: $130 \mathrm{~m}$.

Sustrato: fondo con restos de coral.

\section{Grupo SCLERAXONIA}

Familia ANTHOTHELIDAE Broch, 1916

Género Diodogorgia, Kükenthal, 1919

\section{Diodogorgia nodulifera (Hargitt, 1901)}

Localidad: sur de la isla La Tortuga (MOBR I-767, I-794, I-795).

Descripción: colonias ramificadas en forma arbustiva, de color rojo intenso, de 14 a $17 \mathrm{~cm}$ de alto. El eje está constituido por escleritos rojos no fusionados entre sí. Las ramas terminales tienen un diámetro de 4,5 mm. Pólipos translúcidos y retráctiles.

Profundidad: $130 \mathrm{~m}$.
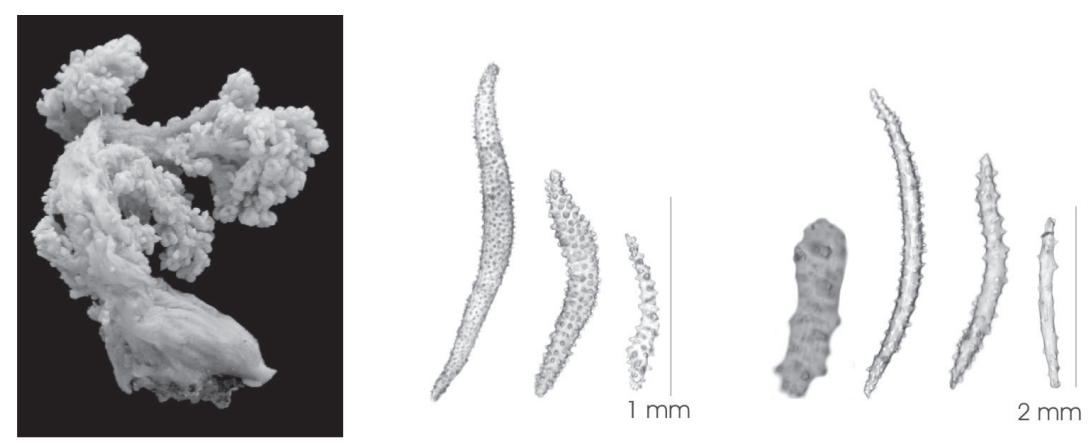

Neospongodes portoricensis

Figura 2. Colonia y escleritos del octocoral de aguas profundas Neospongodes portoricencis.

Figure 2. Colony and sclerites of deep-waters octocoral Neospongodes portoricencis.

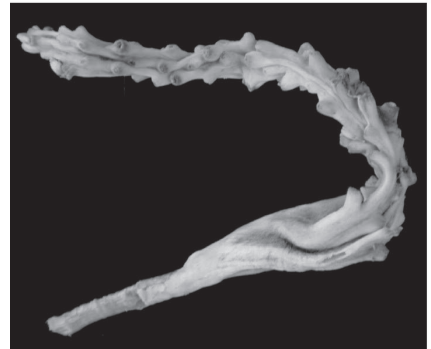

Clavularia sp.

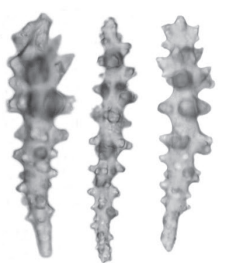

$0,1 \mathrm{~mm}$

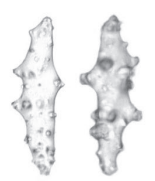

$0,08 \mathrm{~mm}$

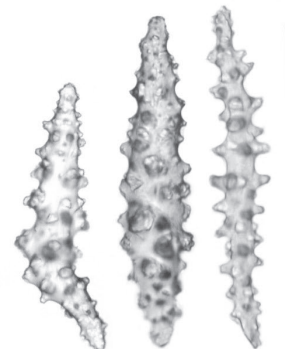

$0,5 \mathrm{~mm}$

Figura 3. Colonia y escleritos del octocoral de aguas profundas Clavularia sp.

Figura 3. Colony and sclerites of deep-waters octocoral Clavularia sp. 
Sustrato: fondo con restos de coral.

Suborden HOLAXONIA Studer, 1887

Familia PLEXAURIDAE Gray, 1859

Género Hypnogorgia Duchassaing \& Michelotti, 1864

Hypnogorgia pendula Duchassaing \& Michelotti, 1864

Localidades: isla La Blanquilla (MOBR I-066; I095), sur de la isla La Tortuga (MOBR I-796).

Descripción: colonia arbustiva ramificada en forma alternada, con crecimiento en un solo plano y $30 \mathrm{~cm}$ de altura. Presenta ramas anastomosadas, un diámetro terminal de 1,1-1,2 $\mathrm{mm}$. Cuando la colonia está en seco, se pueden notar los escleritos, ya que su tamaño puede alcanzar los 0,5 $\mathrm{mm}$ de longitud, éstos están de manera paralela al eje. Los escleritos predominantes son los husos, aunque también se pueden observar bastones rectos y curvos. Los cálices permanecen proyectados a lo largo de toda la colonia con escleritos prominentes formando un arco. En seco, la colonia es muy frágil y quebradiza, presentando un color café oscuro.

Profundidad: 50-130 m.

Sustrato: en fondos rocosos como en fondos con restos de coral.

Género Swiftia Duchassaing \& Michelotti, 1864

Swiftia exserta (Ellis \& Solander, 1786)

Localidad: isla La Blanquilla (MOBR I-065).

Descripción: colonia ramificada con crecimiento en un solo plano, de $22 \mathrm{~cm}$ de alto. Las ramas de la colonia son más largas y separadas que en el género Hypnogorgia. La apariencia externa de la colonia le proporciona un aspecto de cuerpo mullido y esponjoso; sin espículas visibles como en el género Hypnogorgia. Los cálices se destacan como receptáculos amplios y más altos que el diámetro de las ramas terminales (1,0-1,2 mm). En seco, la colonia es blanquecina, en húmedo es de color crema y en vivo es de color rojo-ladrillo.

Profundidad: $53 \mathrm{~m}$.

Sustrato: fondo rocoso.

\section{Swiftia sp.}

Localidad: norte de la península de Macanao (MOBR I-522).

Descripción: colonia ramificada principalmente en un solo plano, algunas de las cuales se curvan hacia arriba. Los pólipos son grandes y cónicos relativamente distanciados entre sí, y no forman filas definidas en los bordes de las ramas. La mayoría de las ramas termina con la presencia de dos pólipos opuestos. El antostele es de forma cónica; el antocodio es alto, posee numerosas filas de espinas y conforma una corona de bastones curvados. El cenenquima contiene escleritos en forma de husos alargados pequeños, además de múltiples cabrestantes.

Profundidad: $60 \mathrm{~m}$.

Sustrato: fondo rocoso.

Género Thesea Duchassaing \& Michelotti, 1860

Thesea guadalupensis Duchassaing \& Michelotti, 1864 (Fig. 4)

Localidad: norte de la península de Paria (MOBR I-734, I-769).
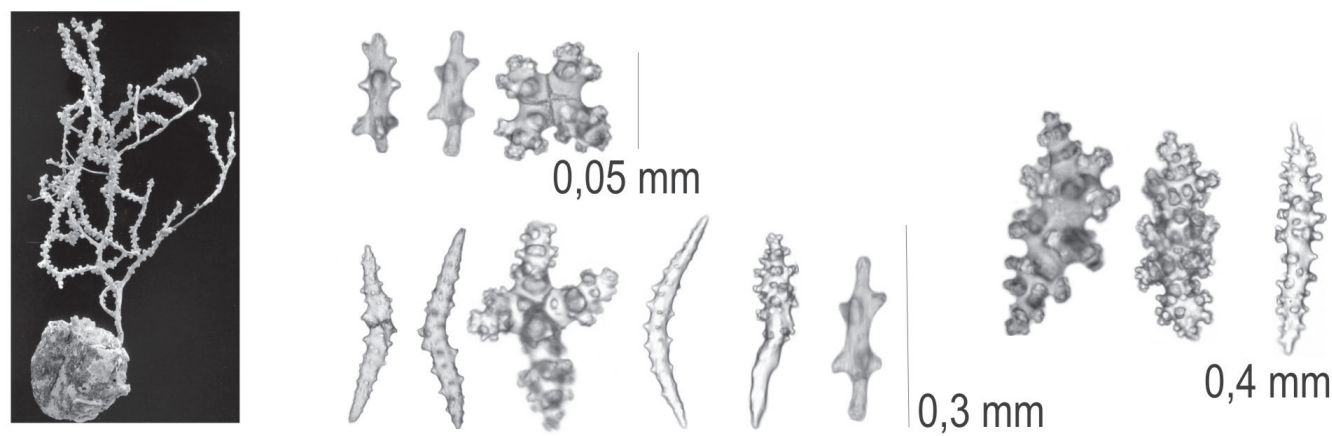

Thesea guadalupensis

Figura 4. Colonia y escleritos del octocoral de aguas profundas Thesea guadalupensis.

Figura 4. Colony and sclerites of deep-waters octocoral Thesea guadalupensis. 
Descripción: colonia ramificada irregularmente, los cálices son prominentes, anchos y cónicos, dispersos en forma azarosa en la superficie de las ramas, pueden agruparse algunas veces hasta cuatro hacia las puntas de las ramas. Los escleritos de la capa externa son husos gruesos y romos de hasta $0,4 \mathrm{~m}$ y también se encuentran en menor densidad cuerpos con cuatro radios, los escleritos de la capa interna son, en general, más pequeños de hasta $0,3 \mathrm{~mm}$. La colonia en húmedo, es de color ocre.

Profundidad: $50 \mathrm{~m}$.

Sustrato: fondo rocoso.

Género Plexaurella Valenciennes, 1855

Plexaurella fusifera Kunze, 1916

Localidad: isla La Blanquilla (MOBR I-098).

Descripción: colonia con ramificación dicotómica de color café claro y de $34 \mathrm{~cm}$ de altura. Las ramas terminales se asemejan a las de $P$. nutans, aunque son más delgadas, con un diámetro de 9 a $10 \mathrm{~mm}$. Presentan una apertura calicular levemente levantada de la superficie de la colonia formando una especie de anillo. Los cálices se encuentran bien separados.

Profundidad: $40 \mathrm{~m}$.

Sustrato: fondo rocoso rodeado de sedimento muy fino.

Familia GORGONIIDAE Lamouroux, 1812

Género Leptogorgia Milne Edwards \& Haime, 1857

\section{Leptogorgia virgulata}

Localidad: norte de la península de Paria (MOBR I-733, I-735, I-792).

Descripción: colonia que en seco es de coloración naranja oscuro, ramificada en un solo plano en forma pinnada, con una altura promedio de $15 \mathrm{~cm}$. Los cálices son altos y están dispuestos en forma bilateral. Los escleritos, tipo husos de la corteza, llegan hasta los $0,15 \mathrm{~mm}$ de longitud y los escleritos son de tipo cabrestantes; los escleritos del pólipo miden de 0,09 a $0,1 \mathrm{~mm}$ y son de color naranja en todo la colonia. Profundidad: $50 \mathrm{~m}$.

Sustrato: fondo rocoso.
Suborden CALCAXONIA

Familia ELLISELLIDAE Gray, 1859

Género Ellisella Gray, 1858

Ellisella barbadensis (Duchassaing \& Michelotti, 1864)

Localidad: isla La Blanquilla (MOBR I-067, I-089, I-168).

Descripción: colonia con ramas muy largas de hasta $86 \mathrm{~cm}$ de altura, las ramas no son muy abundantes y poseen un diámetro de 1,0-1,5 mm. La colonia crece en forma de flagelo. Los cálices son blanquecinos, prominentes y se desarrollan hacia arriba con una disposición biserial.

Profundidad: 50-60 m.

Sustrato: fondo rocoso.

Ellisella elongata (Pallas, 1766)

Localidad: isla La Blanquilla (MOBR I-039).

Descripción: colonia muy flexible, con crecimiento en forma de látigo, con un eje único de $80 \mathrm{~cm}$ de largo y 1,5 mm de diámetro en su sección terminal. Cuando es preservada en húmedo es de color anaranjado y los cálices son un poco más claros y se proyectan hacia arriba.

Profundidad: $47 \mathrm{~m}$.

Sustrato: fondo rocoso.

\section{Género Nicella Gray, 1870}

Nicella guadalupensis Duchassaing \& Michelotti, 1860

Localidad: sur de la isla La Tortuga (MOBR I797).

Descripción: colonias con las ramas aparentemente dicotómicas dispuestas en un solo plano, el diámetro de las ramas es de 0,8 a 1,0 mm y la altura de $11 \mathrm{~cm}$. Los cálices hemisféricos están bien separados y son de color blanco. La colonia en alcohol es anaranjada y en vivo el color es más intenso.

Profundidad: $130 \mathrm{~m}$.

Sustrato: fondo con restos de coral.

Orden PENNATULACEA Verrill, 1865

Suborden SESSILIFLORAE Kükenthal, 1915

Familia RENILLIDAE Gray, 1860 


\section{Género Renilla Lamarck, 1816}

Renilla reniformis Pallas, 1766

Localidad: plataforma Deltana, norte de la península de Paria (MOBR I-799).

Descripción: colonia de forma acorazonada, sin ramificaciones, y se sostiene de un pedúnculo de $1,8 \mathrm{~cm}$ de longitud. La colonia es de color púrpura claro y sus dos antocodios son de coloración más clara.

Profundidad: $90 \mathrm{~m}$.

Sustrato: fangoso.

\section{Familia KOPHOBELEMNIDAE Gray, 1860}

Género Sclerobelemnon Kölliker, 1972

\section{Sclerobelemnon theseus Bayer, 1959}

Localidad: plataforma Deltana, norte de la península de Paria (MOBR I-798).

Descripción: colonia alta, elongada y esbelta de un solo eje con una altura media de $13 \mathrm{~cm}$. Presenta dos secciones a lo largo del eje, la primera parte es fina de unos $0,8 \mathrm{~cm}$ de diámetro, con la que se fija al sustrato blando, en esta parte no posee pólipos; la segunda parte es de un diámetro mayor llegando a $1,5 \mathrm{~cm}$. Aquí los pólipos (no retráctiles) están bien separados y sus cálices son en apariencia obesos, de $5 \mathrm{~mm}$ de diámetro aproximadamente. La colonia es de coloración blanca a pardo.

Profundidad: $90 \mathrm{~m}$.

Sustrato: fangoso.

\section{DISCUSIÓN}

La mayoría de los ejemplares analizados se encontró en sustratos sólidos y consolidados, formados por rocas de origen calcáreo, restos de esqueletos coralinos y conchas de bivalvos. Sin embargo, las especies Renilla reniformis y Sclerobelemnon theseus de las familias Sessiliflorae y Kophobelemnidae respectivamente, se colectaron en su totalidad en sustratos fangosos y arenosos.

De las especies identificadas, tres constituyen nuevos registros para la fauna de octocorales de Venezuela: Neospongodes portoricencis, Clavularia sp. y Thesea guadalupensis, pertenecientes a las familias Nephtheidae, Clavularidae y Plexauridae respectivamente. Las demás especies ya habían sido descritas como fauna de aguas someras por varios autores (Bayer, 1961; Ramírez \& González, 1974; Romero \& Rada, 1999; Losada \& Pauls, 2000; Ra- mírez, 2001; Rada \& Ruiz, 2002).

La bibliografía sobre octocorales de aguas profundas del Caribe sur es muy escasa y está limitada a citas esporádicas dentro de estudios con diferentes enfoques ecológicos o productos de expediciones realizadas en diversas localidades (Mosquera, 1991; Díaz, 1995; Sánchez, 1998). Algunos autores consideran que los octocorales de aguas profundas pertenecientes al orden Pennatulacea y al grupo Stolonifera y Alcyoniina del orden Alcyonacea, son anfiatlánticas y sólo algunas especies son probablemente endémicas del Atlántico americano, incluyendo el Caribe. Dentro de la familia Plexauridae, el género Swiftia, también se considera anfiatlántico. La familia Nephtheidae, a la cual pertenece el género Neospongodes, no se encuentra en aguas someras del Atlántico occidental; sin embargo, es muy común en arrecifes someros del Indo-Pacífico. El género Clavularia pertenece al suborden Stonolifera, el cual es escaso en esta región, habiendo sido citadas solo dos especies en aguas profundas (Deichmann, 1936; Bayer, 1961, 1981). Por su parte, el género Sclerobelemnom suele encontrarse a lo largo del Caribe, usualmente a grandes profundidades que pueden superar los $4000 \mathrm{~m}$ (Williams, 1995).

El oriente de Venezuela tiene gran importancia biogeográfica en cuanto al tipo de fauna que posee debido a sus peculiaridades oceanográficas. Esta zona está influenciada por la afluencia de aguas provenientes de varios ríos, entre los que destacan el Orinoco y el Amazonas (Muller-Karger \& Varela, 1990), y por surgencias estacionales que proveen de aguas frías ricas en nutrientes a la región (MullerKarger et al., 2001; Astor et al., 2003), afectando de manera diferente a los distintos taxa presentes (Capelo \& Buitrago, 1998; Romero \& Rada, 1999). Entre otros efectos, esos fenómenos pueden contribuir con la presencia de especies de amplia distribución latitudinal, lo cual suele ser una característica comúnmente observada en latitudes altas (Floeter \& Soares-Gomes, 1999).

Los fondos profundos con presencia de parches de sustrato duro, como aquellos donde pueden habitar diversas especies de octocorales, son importantes desde el punto de vista ecológico como áreas de relativa alta diversidad en un universo rodeado de fondos blandos, al crear zonas de forrajeo, protección y unidades que favorecen las agregaciones durante la reproducción de peces de interés económico (Turgeon et al., 2002; Swanson, 2004). Esto es de particular importancia cuando estos sustratos están situados en una extensa zona dominada por fondos 
blandos (Ludwig \& Walton, 1957; Guzmán-Alvis \& Díaz, 1993), llegando en algunos casos a ser consideradas áreas marinas protegidas (Turgeon et al., 2002). La presencia y abundancia de ciertas especies de octocorales en aguas profundas y su relación con los fondos duros podría ser utilizada como indicador de áreas con potencial para ser protegidas debido a su posible vinculación con la producción pesquera.

\section{REFERENCIAS}

Álvarez, A. 1981. Estudio de algunos factores de mortalidad de octocorales del parque nacional Morrocoy, Estado Falcón. Tesis de Licenciatura en Biología. Universidad Central de Venezuela, Caracas, 103 pp.

Astor, Y., F. Muller-Karger \& M. Scranton. 2003. Seasonal and interannual variation in the hydrography of the Cariaco basin: implications for basin ventilation. Cont. Shelf Res., 23: 125-144.

Bayer, F.M. 1961. The Octocorallia of west Indian region. A manual for marine biologists. The Hauge, Martinus Nijhoff, Netherlands, 373 pp.

Bayer, F.M. 1981. Key to the genera of Octocorallia exclusive of Pennatulacea, with diagnoses of new taxa. Proc. Biol. Soc. Wash., 94(3): 943-947.

Botero, L. 1987. Zonación de octocorales gorgonáceos en el área de Santa Marta y parque nacional Tayrona, costa Caribe colombiana. An. Inst. Inv. Mar., 17: 61-80.

Capelo, J. \& J. Buitrago. 1998. Distribución geográfica de los moluscos marinos en el oriente de Venezuela. Mem. Soc. Cienc. Nat. La Salle, 150: 109-160.

Dahlgren, E.J. 1989. Gorgonian community structure and reef zonation patterns on Yucatan coral reefs. Bull. Mar. Sci., 45(3): 678-696.

Deichmann, E. 1936. The Alcyonaria of the western part of the Atlantic Ocean. Mem. Mus. Comp. Zool., 53: $1-317$

Díaz, M.L. 1983. Estructura ecológica de una comunidad de octocorales en el parque nacional Morrocoy, Estado Falcón. Tesis de Licenciatura en Biología, Universidad Central de Venezuela, 131 pp.

Díaz, J.M. 1995. Zoogeography of marine gastropods in the southern Caribbean. Anew look at provienciality. Caribb. J. Sci., 31(1-2): 104-121.
Duchassaing, P. \& J. Michelotti. 1860. Mémoire sur les coralliaires des Antilles. Mem. Reale Accad. Sci. Torino, Ser. Sec., 19: 56-87.

Fabricius, K. \& P. Alderslade. 2001. Soft corals and sea fans: a comprehensive guide to the tropical shallow-water genera of the Central-West Pacific, the Indian Ocean and the Red Sea. AIMS, Townsville, Australia, $264 \mathrm{pp}$.

Floeter, S.R. \& A. Soares-Gomes. 1999. Biogeographic and species richness patterns of Gastropoda on the Southwestern Atlantic. Rev. Brasil. Biol., 59(4): 567-575.

Fuenmayor, C.A. 1983. Morfología funcional y crecimiento de octocorales en un arrecife del parque nacional Morrocoy, Estado Falcón. Tesis de Licenciatura en Biología. Universidad Central de Venezuela, Caracas, 49 pp.

González, P. 1970. Algunos octocorales de la isla de Margarita, Venezuela. Bol. Inst. Oceanogr. Univ. Oriente, 9(1-2): 79-92.

González, P. 1972. Octocorales de las aguas someras del golfo de Cariaco. Caribb. J. Sci., 12(3-4): 171177.

Guzmán-Alvis, A. \& J.M. Díaz. 1993. Distribución especial de la taxocenosis Annelida-Mollusca en la plataforma continental del golfo de Salamanca, Caribe colombiano. An. Inst. Inv. Mar., 22: 45-59.

Keith, D.E. 1992. Shallow-water gorgonians (Octocorallia) of Roatan, Honduras. Bull. Mar. Sci., 50(1): 212-226.

La Schiazza, J.A. 1985. Morfología funcional y distribución de frecuencias de tamaño de octocorales en relación a un gradiente de profundidad, en un arrecife coralino del parque nacional Archipiélago de los Roques. Tesis de Licenciatura en Biología. Universidad Central de Venezuela, Caracas, 72 pp.

Lewis, J.C. \& E. Von Wallis. 1991. The function of surfase sclerites in Gorgonians (Coelenterata, Octocorallia). Biol. Bull., 181: 275-288.

Losada, F. \& S.M. Pauls. 2000. Cnidarios. En: M. Aguilera, A. Azócar \& E. González (eds.). Biodiversidad en Venezuela, CONICIT Fundación Polar, Caracas, $37 \mathrm{pp}$.

Ludwig, J.C. \& W.R. Walton. 1957. Shelf-edge calcareous brominences in northeastern Gulf of Mexico. Bull. Amer. Assoc. Petrol. Geol., 41: 2054-2101. 
Márquez, L.M., F. Losada \& M. Rodríguez. 1997. Zonation and structure of a gorgonian community in Venezuela. Proceedings of the 8th Coral Reef Symposium, Panamá, 1: 447-450.

Mosquera, E. 1991. Contribución al conocimiento de la autoecología del octocoral Plexaura homomalla en la isla de la Blanquilla. Tesis de Licenciatura en Biología. Universidad Simón Bolívar, Caracas, $165 \mathrm{pp}$.

Müller-Karger, F. \& R. Varela. 1990. Influjo del río Orinoco en el mar Caribe: observaciones con el CZCS desde el espacio. Mem. Soc. Cienc. Nat. La Salle, 49/50(131-134): 361-390.

Müller-Karger, F., R. Varela, R. Thunell, M. Scranton, R. Boher, G. Taylor, J. Capelo, Y. Astor, E. Tappa, T-Y. Ho \& J.J. Walsh. 2001. Annual cycle of primary production in the Cariaco Basin: response to upwelling and implications for vertical export. J. Geophys. Res., 106(C3): 4527-4542.

Rada, M. 1997. Estudio de la relación simbiótica entre un alga clorofícea y dos especies de gorgonáceos. Tesis de Magíster en Ciencias Biológicas, Universidad Simón Bolívar, Caracas, 161 pp.

Rada, M. \& F. Losada. 1998. Relación simbiótica entre Entocladia endozoica (Ulvales) y Plexaura flexuosa (Gorgonacea: Plexauridae). Rev. Biol. Trop., 46(5): 101-110.

Rada, M. \& L. Ruiz. 2002. Octocorales de la isla La Blanquilla. Dependencias Federales. Memoria. Fundación La Salle de Ciencias Naturales, 157: 31-52.

Ramírez, P. 1978. Fauna coralina de la isla de Cubagua, Venezuela. Tesis de Licenciatura en Biología. Universidad de Oriente, Núcleo Nueva Esparta, 74 pp.

Ramírez, P. 1997. Fauna coralina de los islotes Caribe y Los Lobos, Venezuela. En: P. Ramírez (ed.). Islotes Caribe y Los Lobos. Gobernación del Estado Nueva Esparta, La Asunción, 270 pp.

Recibido: 18 mayo 2005; Aceptado: 12 octubre 2006
Ramírez, P. 2001. Corales de Venezuela. Consejo de Investigación de la Universidad de Oriente. Estado Nueva Esparta, 220 pp.

Ramírez, P. \& P. González. 1974. Ecología y distribución de los octocoralarios de la isla de Coche, Venezuela nororiental. Bol. Soc. Ven. Cien. Nat., 31(128/129): 33-62.

Rodríguez, G. 1959. The marine communities of Margarita Island, Venezuela. Bull. Mar. Sci. Gulf Caribb., 9(3): 238-280.

Romero, N. \& M. Rada. 1999. Octocorales de las aguas costeras de la isla de Margarita, Estado Nueva Esparta, Venezuela. Memoria. Fundación La Salle de Ciencias Naturales, 151(49): 95-115.

Sánchez, J.A. 1998. Black coral-octocoral distribution patterns on Imelda Bank, a deep-water reef, Colombia, Caribbean Sea. Bull. Mar. Sci., 65(1): 215-225.

Stiasny, G. 1941. Gorgonaria von Venezuela (inseln Blanquilla und Los Frailes). Arch. Neerl. Zool., 6: 101-116.

Swanson, C.A. 2004. Effect of substrate availability and conspecific cues on communal oviposition in the apple murex snail Phyllonotus pomum. Mar. Ecol. Progr. Ser., 275: 175-184.

Turgeon, D.D., R.G. Asch, \& B.D. Causey. 2002. The state of coral reef ecosystems of the United States and Pacific freely associated states: 2002. National Oceanic and Atmospheric Administration/National Ocean Service/National Centers for Coastal Ocean Science, Silver Spring, 265 pp.

Williams, G.C. 1995. Preliminary assessment of the phylogenetics of pennatulacean octocorals, with a reevaluation of Ediacaran frond-like fossils, and a synthesis of the history of evolutionary thought regarding the sea pens. Proceedings of VI International Conference of Coelenterate Biology, pp. 497-509. 